\title{
Acoustic detection of ultra-high energetic neutrinos - a snap shot -
}

\author{
Rolf Nahnhauer
}

DESY, Platanenallee 6, D-15738 Zeuthen, Germany

\begin{abstract}
Already more than 30 years ago the acoustic particle detection method has been considered to be one possibility to measure signals from ultra-high energetic neutrinos. The present status and problems of corresponding model predictions are discussed in comparison with existing experimental measurements. Available acoustic sensors and transmitters are described and new ideas for corresponding applications are mentioned. Different methods for in-situ calibrations are discussed. Results of measurements of acoustic test arrays at different sites are presented in some detail. Future activities for applications of the technology in large size detectors are evaluated.
\end{abstract}

Keywords: acoustic neutrino detection, cosmogenic neutrinos, acoustic transducers PACS: 43.58, 43.60Fg, $95.85 \mathrm{Ry}$

\section{Introduction}

The detection and study of ultra-high energetic neutrinos above $10^{17} \mathrm{eV}$ created in cosmic sources or by interaction or decay of even higher energetic particles became of increasing interest during the last decade. Several interesting questions of particle physics, astrophysics and cosmology, could be studied measuring interactions of such neutrinos on Earth with reasonable statistics [1].

Corresponding neutrino flux predictions were already small ten years ago [2] but had to be decreased further by new bounds from cosmic ray [3] and high energy gamma ray measurements [4]. Present day experiments could derive therefore until now only flux limits [5, 6, 7, 8]. A first detection of such neutrinos is expected with detectors of $\sim 100 \mathrm{~km}^{3}$ size. To get reasonable statistics will need probably about an order of magnitude larger detector volumes. It seems impossible today to instrument such experiments with conventional optical detectors within reasonable cost limits. Acoustic particle detection may be one option among others to overcome this problem [9].

The possibility to detect charged particles by the sound they produce passing through matter was the first time mentioned in 1957 by G. Askaryan [10]. About 50 20 years later a corresponding model was formulated
[11, 12], and first ideas about a $100 \mathrm{~km}^{3}$ acoustic detector were discussed seriously [13]. In the following sections it will be shown, how far the predictions of the Thermo-acoustic Model could be confirmed experimentally and what questions have still to be answered.

During the last 10 years different groups tried to use acoustic test arrays to extract basic information needed to build large scale detectors in different materials and environmental conditions. Their results will be summarized in the second part of this paper. Finally future steps for the improvement of the acoustic technology and its application will be mentioned.

\section{The Thermo-Acoustic Model}

Ideas about the Thermo-acoustic Model of the creation and propagation of sound in particle interactions were displayed for the first time at the 1976 DUMAND meeting [14]. They were formulated independently by Bowen [12] and Askaryan and Dolgoshein [11]. More detailed descriptions of both concepts were published three years later [15, 16]. Recently a new approach based on [16] has been published including signal attenuation effects [17].

In neutrino interactions a charged or neutral lepton and a hadronic particle cascade is produced. The cascade gives rise to a large energy deposition in a small 


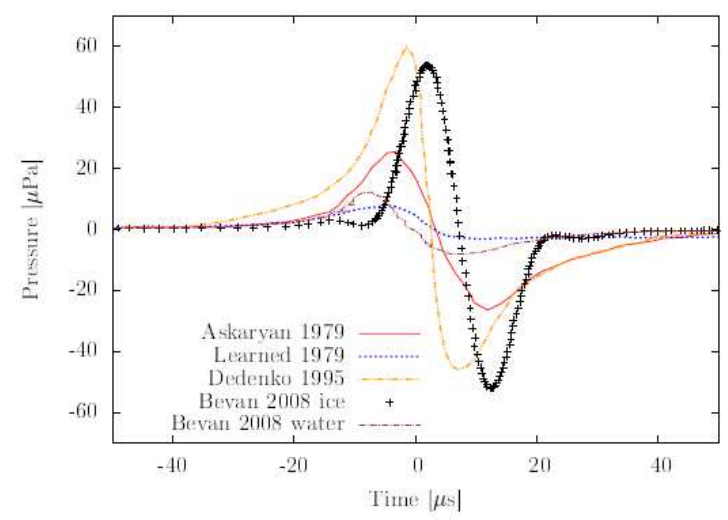

Figure 1: Signal strength from three different acoustic model parameterizations [18] and inclusion of attenuation effects [17] (from [19])

volume in a very short time. The volume is overheated what gives rise to a pressure wave which developes orthogonal to the cascade and therefore the incident neutrino direction.

\section{1. model predictions}

An illustrative way to describe the dependencies of the important quantities and variables of the process is given in [15].

$$
\begin{aligned}
p & =\left(k / c_{p}\right)(E / R) M \\
M & =\left(f^{2} / 2\right)(\sin x / x) \\
f & =v_{s} /(2 d) \\
x & =(\pi L / 2 d)(\sin \delta)
\end{aligned}
$$

with $p$ : pressure amplitude, $E$ : cascade energy, $R$ : distance to receiver, $f:$ frequency, $v_{s}:$ speed of sound, $d$ : cascade diameter, $k$ : volume expansion coefficient, $c_{p}$ : specific heat, $L:$ cascade length, $\delta:$ angle between normal to cascade direction and receiver.

The model predicts a linear dependence of the pressure amplitude on the particle cascade energy, which is related to the incoming neutrino energy and allows to determine the neutrino direction from measuring the pressure wave propagation through the medium.

Unfortunately absolute signal predictions are uncertain mainly due to the not well known particle interaction and energy loss process at ultra-high energies. Different assumptions about what values should be used for the width and the length of a cascade of a certain energy 106 give rise to signal predictions which differ by about an 107 order of magnitude (see fig. 1).

In the considered energy range two other phenom- 109 ena have also to be taken into account: the Landau- ${ }_{110}$
Pomeranchuk-Migdal-effect and photo- and electronuclear reactions [20]. The LPM-effect predicts decreasing cross section for bremsstrahlung and pair production, elongating hadronic cascades above about $10^{18}$ $\mathrm{eV}$ whereas the second effect act in the opposite direction at energies above $10^{20} \mathrm{eV}$.

\section{2. target material effects}

One could rewrite eq. 1 in the form

$$
p=\gamma(E / R) M^{\prime}
$$

with $\gamma=v_{s}^{2}\left(k / c_{p}\right)$ and $M^{\prime}=(1 / 2)\left(1 / d^{2}\right)(\sin x / x)$.

The Grueneisen coefficient $\gamma$ is a strongly material dependent quantity. In tab. 1 this is displayed for three materials under discussion for acoustic detector applications. At the same incoming energy signals in ice should be therefore nearly an order of magnitude larger than those in water. In salt even larger signals are expected. Also permafrost was recently suggested to give rise to quite large signals [22].

Table 1: Thermo-acoustic model parameters and boundary conditions for three different materials (adapted from [21])

\begin{tabular}{|l|c|c|c|}
\hline & water & South Pole ice & salt \\
\hline \hline$c(\mathrm{~m} / \mathrm{s})$ & 1530 & 3880 & 4560 \\
\hline$\left(k / 10^{-5}\right)\left[\mathrm{K}^{-1}\right]$ & 25.5 & 12.5 & 11.6 \\
\hline$c_{p}\left[\mathrm{~J}(\mathrm{~K} \mathrm{~kg})^{-1}\right]$ & 3900 & 1720 & 839 \\
\hline$\gamma$ & 0.153 & 1.12 & 2.87 \\
\hline$f_{\max }[\mathrm{kHz}]$ & 7.7 & 20 & 42 \\
\hline refraction & moderate & very small & small? \\
\hline$\lambda_{\text {att }}$ & $>1000 \mathrm{~m}$ & $\sim 300 \mathrm{~m}$ & $>100 \mathrm{~m}$ \\
\hline noise & variable & stable, $<14 \mathrm{mPa}$ & small? \\
\hline
\end{tabular}

For water things are even more complicated, because the volume expansion coefficient for water depends strongly on the water temperature. At 4 degree Celsius it is equal to zero having different signs below and above this temperature. The signal strength for acoustic pulses in water depends therefore strongly on the specific location of the neutrino interaction and may vary also by a factor 10 (see [23, 24]).

\section{Experimental verifications}

Already at the end of the seventies of the last century several experiments were performed to test the predictions of the Thermo-acoustic Model using acceleratoror laser beams [24, 25]. The basic experimental arrangement is shown in fig. 2. 

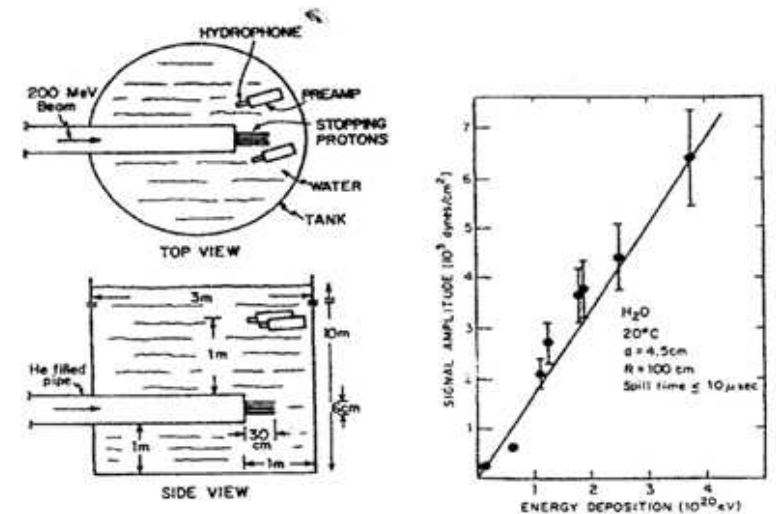

Figure 2: Experimental setup and results for signal dependence on deposited energy for an early Thermo-acoustic model check (from [24])

An intense laser or low energy proton beam is stopped in a water tank. Varying the beam parameters allowed several model checks like the following:

- varying intensity

(E-dependence)

- varying beam diameter $\quad(f$-dependence $)$

- varying distance $\quad(R$-dependence $)$

- varying liquids $\quad(\gamma$-dependence $)$

During the last decade several similar tests have been made by different groups for water [26, 27, 28], ice [29, ${ }_{150}$ 30] and permafrost [22]. The general conclusions from ${ }_{151}$ all these tests are the following:

- many predictions of the Thermo-acoustic Model could be confirmed

- the dominant mechanism for acoustic signal production is thermal expansion

- other contributions, e.g. from micro-bubble forma- ${ }^{15}$ tion could not completely be excluded

- absolute signal values could not be checked, because the energy deposit in the different tests was difficult to calculate precisely and was different to the ultra-high energetic particle case.

An unsolved problem was until recently also the rel- ${ }_{164}$ ative signal strength in different target materials. It 165 is therefore very valuable that an experiment is per- 166 formed this year at the Aachen-Acoustic-Laboratory 167 aiming to answer this question [31]. A well controlled 168 laser beam is stopped in a large tank placed in an even 169 larger freezer. This allows measurements in a tempera- 170 ture range from +20 to -25 degree Celsius in water and 171 ice. In fig. 3 acoustic signals for both configurations are 172 shown. The time difference of the signals is due to the 173
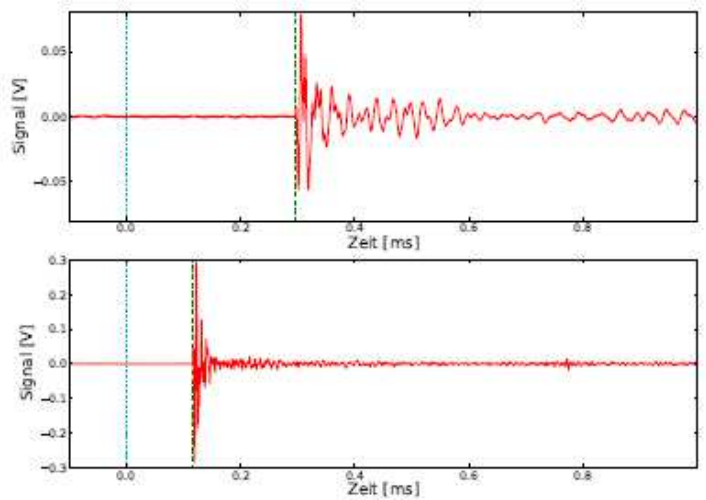

Figure 3: Acoustic signals from a laser beam shot in a tank filled with water (top) and ice (bottom) (from [31])

different velocity of sound in both materials. The signal strength in ice is about a factor six larger than in water consistent with the theoretical expectation. The exact comparison has however to be done for the corresponding pressure amplitudes. More detailed results of these measurements will be available soon.

\section{Acoustic transducers}

Acoustic transducers for ultra-sound have been developed since long for applications in water. Their quality profited from their use in military projects. Today commercial products are available from several companies. Special requirements like stability at high pressure for use at large water depth lead to considerably high prices per piece. Because large numbers of corresponding devices are necessary to build large detector arrays, several attempts were started to build own sensitive sensors and transmitters. For applications in ice, salt and permafrost this was unavoidable anyway.

\section{1. piezo ceramic sensors}

Nearly all acoustic sensors in use in todays test arrays are based on piezo-ceramic elements [32]. Within the AMADEUS project a nice comparison of different commercial and self-made devices has been performed [33]. In fig. 4the response of three of their sensors to the same transmitter signal is shown. An interesting development is their "Acoustic Module", where piezo-ceramic elements are integrated in a glass pressure sphere otherwise used to contain photomultipliers for light detection in the ANTARES experiment [34].

The SPATS group connected to the IceCube experiment started their own developments for ice applications with a similar concept [29, 35] but changed their 


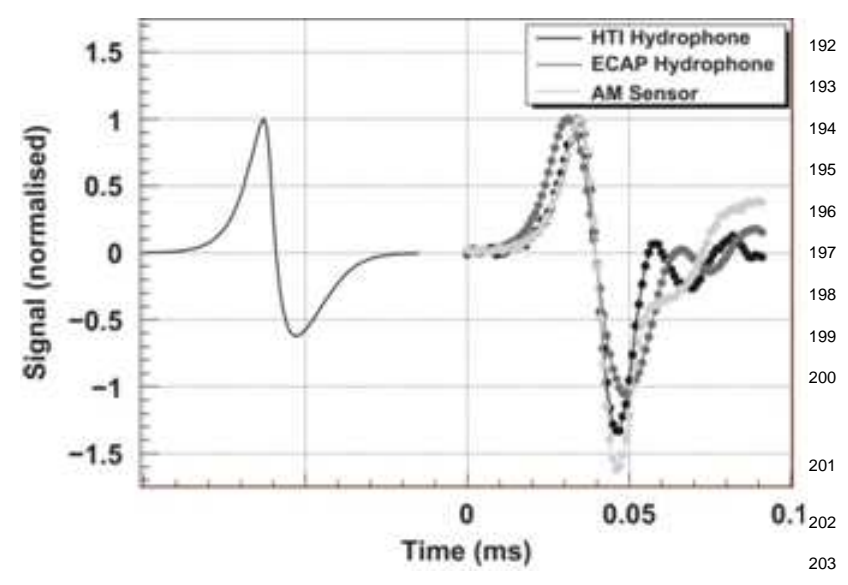

Figure 4: Comparison of the response of three different acoustic sensor types to a bipolar pulse (from [33])

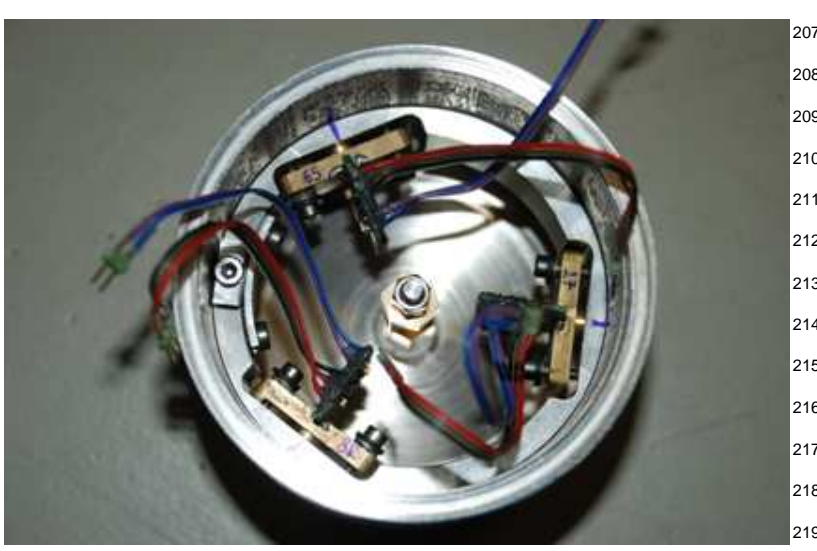

Figure 5: SPATS second generation sensor module (from [36])

design finally to a cylindrical steel pressure housing ${ }^{223}$ where three piezo-ceramics are pressed against the in- ${ }^{224}$ ner wall (see fig. 5). Typical sensitivities of sensors in ${ }^{225}$ use are in the range -190 to $-110 \mathrm{~dB}$ re $1 \mathrm{~V} / \mu \mathrm{Pa}$.

Recently the use of piezo-foils for the design of ${ }^{227}$ acoustic sensors has been reported [31]. This would ${ }^{228}$ have the advantage that one would be more flexible in ${ }^{229}$ sensor shape and size. Results from forthcoming tests ${ }^{230}$ will be hopefully published soon.

\section{2. other sensor concepts}

A "new" concept for acoustic sensors under discussion in particle detection applications since a few years ${ }_{234}$ is the use of acousto-optic hydrophones. In fact the corresponding working principles using either frequency 235 or intensity modulations in optical fibers has been dis- 236 cussed already more than 30 years ago [37].

Recently several groups published results for a fiber ${ }_{238}$ laser, where the modulation of the cavity size by pres- 239 sure pulses leads to modulations of the laser wavelength detectable by interferometry [38]. The concept has been proven to work at a static pressure of $35 \mathrm{MPa}$, i.e. 3500 $m$ depth with higher sensitivity and better resolution than piezo-ceramic based devises. An application in a real open water test array is however still missing.

The application of coupled waveguide intensity modulated hydrophones is under discussion for an upgrade of the BAIKAL acoustic test setup [23].

\section{3. transmitters}

Acoustic transmitters are normally used in acoustic arrays to get position and calibration information. An exceptional idea was realized for this purpose in the SAUND-1 experiment, where light bulbes of different size were deployed on top of seven hydrophones at the AUTEC military array near the Bahamas [39].

The SPATS group published results from different types of pingers fixed in ice or used in water filled holes to measure the acoustic attenuation length in South Pole ice [40]. A byproduct of these measurements was the observation of shear waves in the ice [41].

Position monitoring is also a problem of all deep water optical neutrino telescopes. It is normally solved by fixing strong acoustic transmitters at the sea bed and by adding some hydrophones to the optical strings. Pulses emitted with short time differences allow then precise position monitoring at the $10 \mathrm{~cm}$ scale [42]. For the planned KM3Net detector a corresponding system is under development, which will allow to observe pulses from particle cascades with high sensitivity [43].

To test the sensitivity of acoustic arrays in-situ is still an unsolved problem. Two groups try to develop acoustic pulser systems which mimic pulses from ultra-high energetic neutrino interaction in strength and shape. Successful tests have been made using arrays with more than 5 transmitters taking into account the transfer function between emitted and received signals [44]. In another approach a parametric acoustic source is used. The overlay of two high frequency signals fed to an emitter leads to the production of a bipolar pulse at lower frequency whereas the remaining high frequency components are quickly absorbed in the medium [45].

\section{Sensor calibration}

For the application of the acoustic technology for particle detection the use of carefully calibrated sensors is mandatory. Several methods are applied for this purpose in the laboratory [46], e.g. comparison with a calibrated reference hydrophone and reciprocity calibration or use 


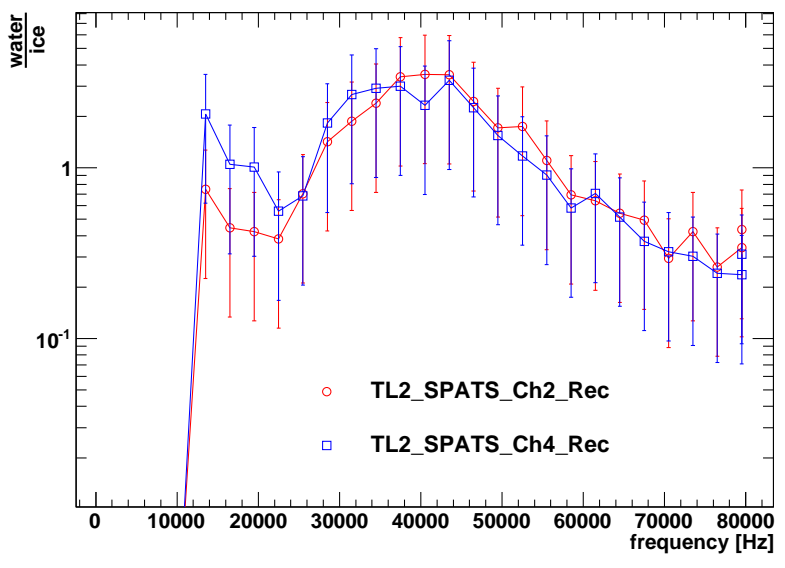

Figure 6: Preliminary result for the ratio of sensitivities of a third generation SPATS sensor deployed in water and ice (from [49]) of calibrated emitters. With the last method all sensors 273 used for the AMADEUS project were calibrated in de- 274 pendence of the azimuthal and zenith angle in a water 275 tank [33]. One has to keep in mind, however, that the 276 sensor sensitivity depends on the specific environmental 277 conditions at the deployment location as e.g. tempera- 278 ture and pressure. Together with a NATO institute the 279 ONDE-group has developed a standard procedure for 280 under pressure calibration. They reported a sensitivity change of about $2 \mathrm{~dB} / 1000 \mathrm{~m}$ water depth [47].

In-situ calibration in ice is even more complicated than in water. Beside pressure effects, deep temperature ${ }_{283}$ and impedance changes between ice and water have to 284 be taken into account. The SPATS group has calibrated 285 their sensors in water and studied separately the depen- 286 dence on pressure (in a water-oil mixture), temperature 287 (in air) and impedance (measuring noise level changes 288 during sensor freeze-in). Assuming that the correspond- 289 ing sensitivity changes can just be multiplied, they got a 290 final result with about 40 percent error [48].

A direct comparison of calibration results in water 282 and ice at normal pressure was done for a third gen- 293 eration SPATS sensor in the Aachen-Acoustic Labora- 294 tory [49]. Preliminary results of a reciprocity calibra- 295 tion, shown in fig. 6 are consistent with the estimation 296 described above. The final result of these measurements 297 is expected to be published soon.

\section{Acoustic test arrays}

During the last decade results were reported from six 301 test sites either using part of military hydrophone arrays 302 or installing own acoustic sensors. The main purpose 303 of these studies was to clarify how sensitive acoustic 304

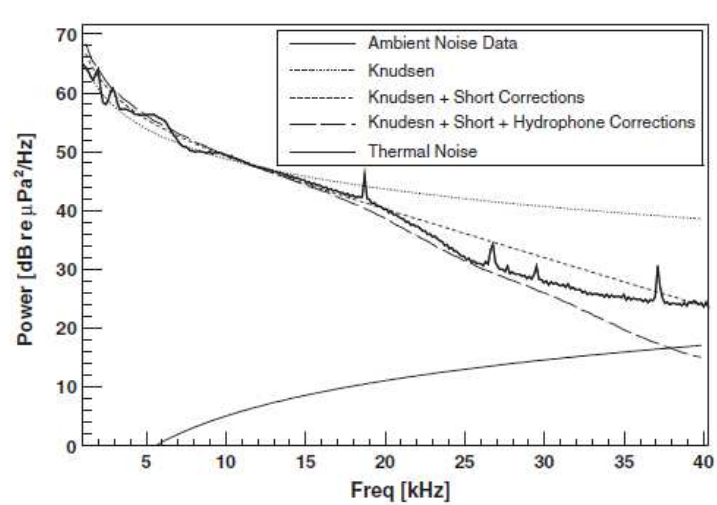

Figure 7: Average ambient noise level measured by SAUND (from [50])

detectors could be to signals from neutrino interactions at given environmental conditions like acoustic noise, signal attenuation and refraction. A few prominent results of the different groups will be discussed below. It is however out of the scope of this presentation to give a detailed overview about presently available data. To get more information the reader is pointed to the references given in this paper and to the proceedings of the ARENA conferences since 2005 [9].

\subsection{SAUND}

The "Study of Acoustic Ultra-high Neutrino Detection - SAUND" started in 2003. Seven hydrophones of the military AUTEC array near the Bahamas were used to search for acoustic signals. In 196 days $65 \times 10^{6}$ triggers were taken in a sensitive volume of about $15 \mathrm{~km}^{3}$. The data were used to calculate the first acoustic neutrino flux limit [39].

In the second phase of this experiment the number of used hydrophones increased to 49 and the volume under study to $1500 \mathrm{~km}^{3}$. 130 days of data taking allowed detailed studies of acoustic noise behaviour (see fig. 7) [50]. In a complex data reduction and signal processing procedure two events were found compatible with showers from neutrino interactions above $10^{22} \mathrm{eV}$. The result was used to derive an improved neutrino flux limit (see fig. 12) [51].
298

299 UK. Eight hydrophones of the RONA military array in North-West Scotland are used for acoustic signal searches. Between 2006 and 2008 nearly $30 \mathrm{~Tb}$ of raw data have been collected. Advanced signal processing 


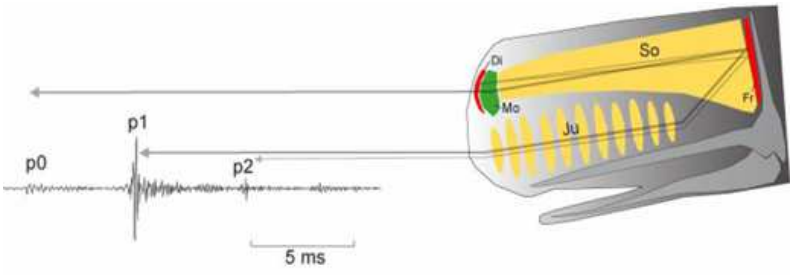

Figure 8: Acoustic signals from a sperm whale (from [47])

and filtering technologies were developed by the group and applied to the data [52, 53]. Corresponding data reduction and efficiency calculations allowed to derive a neutrino flux limit in the same energy range as the SAUND result (see fig. 12). Furthermore information about signal attenuation and localization was collected. The examination of the data for black hole signatures lead to the calculation of a corresponding upper limit [54].

\section{3. $O N D E$}

Within the "Ocean Noise Detection Experiment ONDE" four hydrophones forming a tetrahedron were deployed in 2005 to about $2000 \mathrm{~m}$ depth in the Mediterranean See $25 \mathrm{~km}$ offshore of Catania/Italy. Noise was monitored about five minutes every hour. The average noise level in the $20-43 \mathrm{kHz}$ frequency region was found to be $5.4 \pm 2.2 \pm 0.3 \mathrm{mPa}[55]$. The noise level was strongly correlated with the actual environmental conditions at the sea surface.

Signals observed in the noise data more often than expected were produced by a population of sperm whales. Analyzing these signal in detail (for an example see fig. 8) even allowed to derive age and gender of the animal emitting it [47]. Corresponding studies will continue in ${ }^{344}$ collaboration with marine biologists.

\subsection{Baikal}

A subgroup of the collaboration which constructed ${ }_{347}$ and operates the first optical neutrino telescope in lake ${ }_{348}$ Baikal [56] has deployed a digital hydro-acoustic mod- 349 ule with four hydrophones at a regular tetrahedron of $1.5{ }_{350}$ $\mathrm{m}$ edge length in $150 \mathrm{~m}$ depth at one of the outer strings ${ }_{351}$ of the optical array. With this device and its predeces- ${ }_{352}$ sors extensive environmental studies have been done. ${ }^{353}$ The noise level was measured most of the time below ${ }_{354}$ $5 \mathrm{mPa}$. Accepting only signals from the deep lake one 355 interesting neutrino-like event has been observed (see ${ }_{356}$ fig. 9).

In March 2011 an acoustic string with three acoustic ${ }_{358}$ modules has been deployed [23]. Data taking is ongoing 359
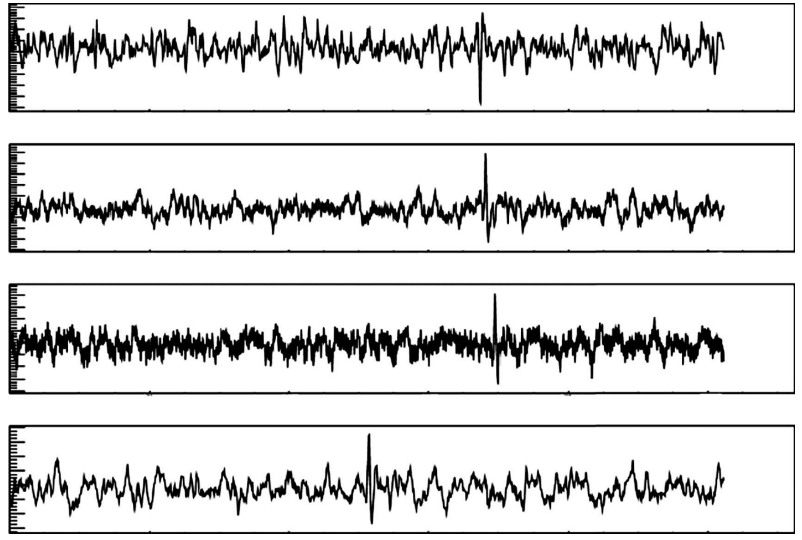

Figure 9: Acoustic event reconstructed as upward going neutrino-like signal (from [23])

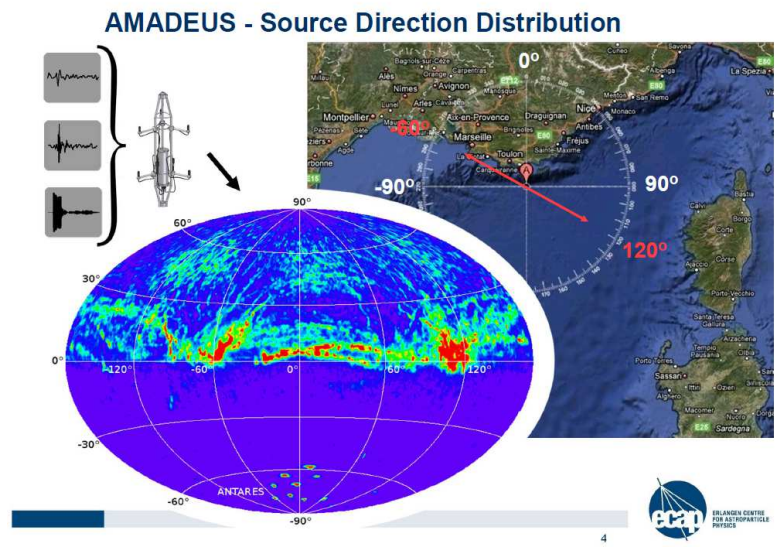

Figure 10: Angular distribution of transient events observed with the AMADEUS detector(from [58])

and first results are expected to be shown at next years conferences.

\subsection{ANTARES}

A detailed description of the design and performance of the "ANTARES Module for the Acoustic DEtection Under the Sea - AMADEUS" can be found in [33]. 36 sensors are located at three storeys of both string 12 and the instrumentation line of the ANTARES optical neutrino telescope [34] located at a depth of $\sim 2500$ $\mathrm{m}$ in the Mediterranean sea about $40 \mathrm{~km}$ distant to Toulon/France. Data taking started in 2007. Extensive noise studies have shown strong correlations to actual weather conditions. A high statistics frequency dependent noise measurement reflects similar results like those found with ONDE in the deep sea near Sicily (see section 6.3). 
The arrival direction of transient acoustic signals could be determined (see fig. 10] [57]. It was found that these signals have mostly antroprogenic origin being due to heavy ship traffic in this region of the sea.

\subsection{SPATS}

The "South Pole Acoustic Test Setup - SPATS" has been deployed in the upper empty part of holes drilled for the IceCube neutrino observatory [5]. Seven acoustic stations with transmitters and receivers are positioned between $80 \mathrm{~m}$ and $500 \mathrm{~m}$ in the ice at the South Pole, with a maximum distance of about $520 \mathrm{~m}$ between strings [36]. Data taking started in early 2007. Since then results have been published for the speed of sound of pressure and shear waves and their refraction versus depth [41], the acoustic attenuation length [40] and the ambient noise level [48].

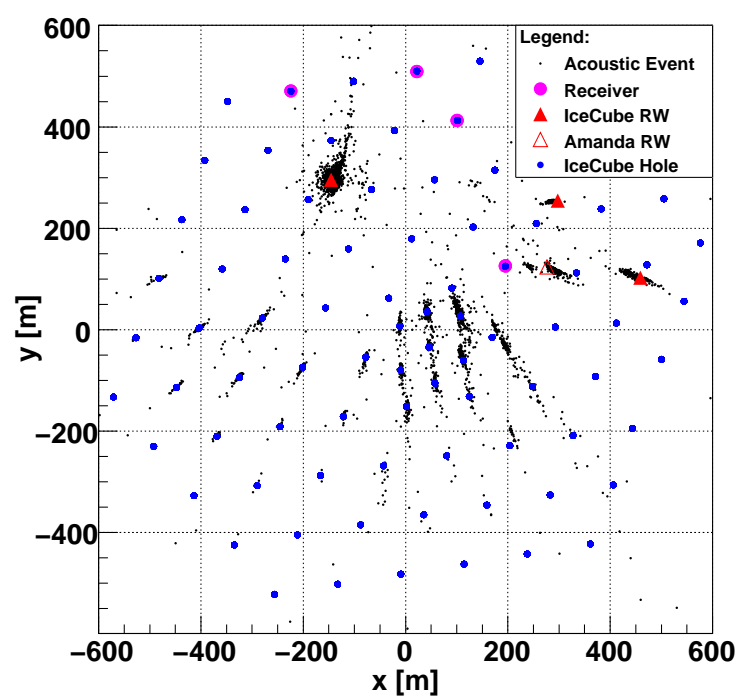

Figure 11: Distribution of transient signal source locations in the $\mathrm{x}$ y plane compared with the position of IceCube holes and Rod-wells (from [48])

Transient events were observed from re-freezing Ice-
Cube holes and from water reservoirs used for hole drilling (see fig. 11). From the non-observation of acoustic signals in a region outside the IceCube construction area a neutrino flux limit has been estimated 405 (see fig.12).

\section{Acoustic neutrino-flux limits}

Neutrino flux limits have been derived until now in 409 three acoustic test experiments [51, 53, 48], all not op- 410 timized or not designed for this purpose. In fig. 12

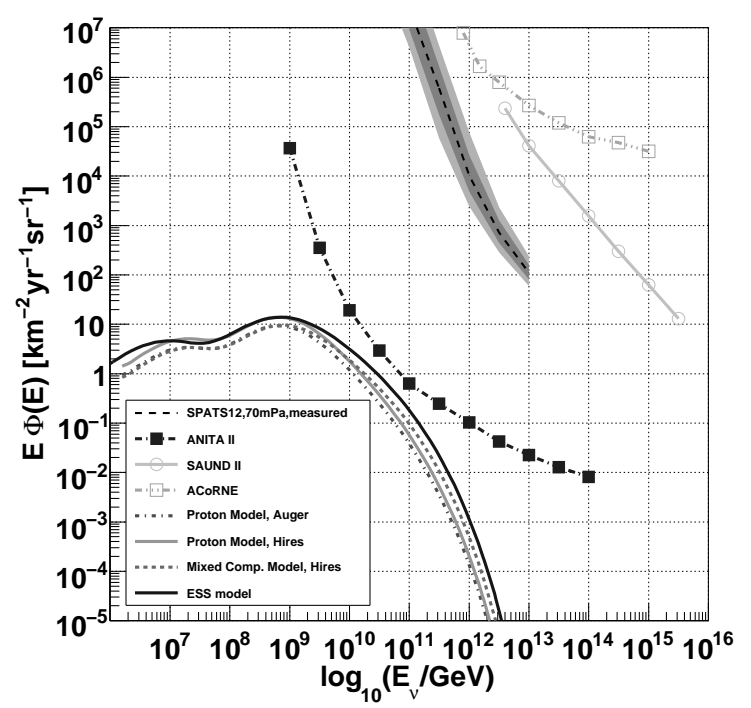

Figure 12: Available neutrino flux limits from acoustic test arrays compared to some model predictions and results from some radio detectors sensitive at highest energies (from [48])

the corresponding results are shown and compared with predictions of one frequently quoted cosmogenic neutrino flux model and its recent modifications [2]. The presently best limit in the considered energy region has been published recently by the ANITA collaboration using radio antennas as payload of a balloon circling in a hight of $35 \mathrm{~km}$ around Antarctica [8]. The detector was searching for pulses of coherent radio emission from neutrino induced cascades in the ice below in a volume of about $1.6 \times 10^{6} \mathrm{~km}^{3}$.

The acoustic neutrino flux limits are today still more than four orders of magnitude less sensitive than the best radio limits which is partly explained by the huge difference in the corresponding detection volumes used. What makes all limits difficult to interpret are the partly unknown systematic errors of the measurements and the reliability of assumptions made for efficiency and sensitivity calculations. This has been discussed e.g.in [48].

\section{Future activities}

The experiments mentioned in section 6 have in the meantime either finished or achieved their primary goals. Follow up programs are under discussion or already in a planning stage.

\section{1. acoustic detection in water}

With the present facilities "fake neutrino sources" (see section 4.3) will be used to get better knowledge 
about in-situ detector efficiencies and trigger schemes. ${ }_{461}$ On an intermediate time scale design studies towards a ${ }^{462}$ $\mathrm{km}^{3}$-sized hybrid acousto-optic detector will hopefully ${ }^{463}$ be followed by its construction in the Mediterranean ${ }_{465}^{464}$ KM3Net project [47]. Most European acoustic activi- 466 ties should converge around this program.

\section{2. acoustic detection in ice}

Ice provides probably the best conditions to ${ }^{471}$ build a hybrid radio-acoustic-optical detector allowing ${ }_{473}^{472}$ background-free detection of the rare neutrino signals 474 above $10^{18} \mathrm{eV}$. Having evaluated the acoustic ice prop- ${ }^{475}$ erties at the South Pole, the boundary conditions for ${ }^{476}$ such a detector are now mostly known at that location. Corresponding Monte Carlo design studies are under 479 way. They show, that already a $100 \mathrm{~km}^{3}$ scale detector ${ }^{480}$ would need a large number of holes to be drilled. The ${ }^{481}$ development of robotic drilling and deployment meth- ${ }_{483}$ ods is mandatory for such a project. First attempts in 484 that direction are just starting [59].

The detection of ultra-high energy neutrinos and the ${ }^{486}$ detailed study of their interactions remains a top priority 488 topic of astroparticle physics also in the long term fu- ${ }^{489}$ ture. Corresponding experiments need most probably to ${ }^{490}$ employ multiple detection methods with different sys- ${ }^{491}$ tematics for successful operation. The acoustic particle ${ }_{493}$ detection technology is qualified to be one of them.

\section{Acknowledgements}

I would like to thank the organizers of this conference for the invitation to give this presentation. I thank also N. Budnev, G. Gratta, D. Heinen, R. Lahmann and 502 G. Riccobene for providing new information about activities of their groups. I am indebted to J. Berder- ${ }_{505}^{504}$ mann for valuable suggestions and critical reading of 506 the manuscript.

\section{References}

[1] R. Nahnhauer, NIM A (2010), doi:10.1016/j.nima.2010.11.010

[2] R. Engel, D.Seckel, T. Stanev,Phys. Rev. D64 (2001) 093010 , update 2008: (ftp:://ftp.bartol.ude.edu/seckel/ess-gzk/2008)

[3] L.A. Anchordoqui et al., Phys. Rev. D76, (2007) 123008

[4] M. Ahlers et al., Astropart.Phys 34, (2010) 106

[5] R. Abbasi et al.,Phys. Rev. D83, (2011) 092003

[6] J. Abraham et al., Phys. Rev. D79, 2009) 102001

[7] I. Kravchenko et al., Phys. Rev. D73, (2006) 082002, update: arXiv 1106:1164

[8] P.W. Gorham et al., Phys. Rev. D82, (2010) 022004

[9] R. Nahnhauer, S. Boeser (eds.), Proceedings of ARENA 2005 Int. J. Mod. Phys. A21 (2006); L.Thompson, S. Danaher (eds.) Proceedings of ARENA2006, J. Phys.Conf. ser. 81, (2007); A Capone et al., (eds.) Proceedings of ARENA2008, NIM A 604
(2009); P. Latridou et al., (eds.) Proceedings of ARENA2010, in Press

[10] G.A. Askaryan, Atomnaja Energiya V3 (1957)152

[11] G.A. Askaryan, B. Dolgoshein, JETP Lett.25 (1977) 213

[12] T. Bowen, Proceedings of the 15th ICRC, Plowdiv, vol.6 (1977)p. 277

[13] V.S. Berezinsky, G.T. Zatsepin, Sov. Phys. Usp.. 20 (1977) 361

[14] A. Roberts, Rev. Mod. Phys. 64 (1992) 259

[15] G.A. Askaryan et al., NIM 164 (1979) 267

[16] J. Learned, Phys. Rev. D 19 (1979) 3293

[17] S. Bevan et al., NIM A607 (2009) 398

[18] A.V. Butkevich et al., Fiz. El. Sch. Atom. Jadr. 29 (1998) V3

[19] F Descamp, Thesis, Universiteit Gent, (2009)

[20] L. Gerhard, S. Klein, Phys. Rev. D82 (2010) 074017

[21] P.B. Price, J. Geophys.Res. 111 (2006) B02201.

[22] R. Nahnhauer et al., NIM A 587 (2008) 29

[23] V. Aynutdinov et al., NIM A doi:10.1016/j.nima 2010.11 .153

[24] L. Sulak et al., NIM 161 (1979)203

[25] R. Golubnichy et al.,Proceedings of DUMAND 1979, p.148

[26] V. I. Abdul et al., Instr. Exp. Techn. 44 (2001) 327

[27] K. Graf et al., Int. J. Mod. Phys. A21 (2006) 127

[28] G. De Bonis et al., NIMA 604 (209) 199

[29] S Boeser, Thesis, Humboldt Universitaet Berlin, 2006

[30] J. Stegmeier, Diploma Thesis, Johannes Gutenberg Universitaet Mainz, 2004

[31] D.Heinen, Diploma Thesis RWTH Aachen, 2011

[32] G. Anton et al., Astropart. Phys. 26 (2006) 301

[33] J.Aguilar et al.,NIM A 626-627 (2011) 128

[34] M. Angeron et al., arXiv:1104.1607 (2011), subm. to NIM A

[35] J. Boeser, Int. J. Mod. Phys. A21 (2006) 107

[36] Y. Abdou et al., arXiv:1105.4339 (2011) subm. to NIM A

[37] J. N. Fields, Appl. Optics, V18, (1979) 3533

[38] P.E. Bagnoli et al., NIMA 567(2006) 515, E. Maccione et al.,NIMA 572 (2007) 490; A.Cotrufo et al. NIMA 604 (2009) 219; J. Wang et al., Optoel. Let. V3 (2007) doi:10.1007/s11801007-6169-1; S. Goodman et al., Proc. SPIE V 7004 (2008), doi:10.1117/12.785937; B.Guan et al. , Opt. Expr. V17 (2009)19544 and references therein

[39] J. Vandenbroucke et al., Astrophys. J. 621 (2005) 301

[40] R. Abbasi et al.,Astropart. Phys. 34 (2011) 382

[41] R. Abbasi et al.,Astropart. Phys. 33 (2010) 277

[42] M. Ardid,NIM A 602 (2009) 174

[43] P. Ameli et al., NIM A 626 (2011) 211

[44] W. Ooppakaev, S. Danaher, NIM A 2011), doi:10.1016/j.nima.2010.11.142

[45] M. Ardid et al., NIM A (2010), doi:10.1016/j.nima.2010.11.139

[46] M. Ardid, NIM A 604 (2009) 203

[47] G. Riccobene, contribution to this proceedings

[48] R. Abbasi et al., arXiv:1103.1216

[49] L. Paul, RWTH Aachen internal report, 2011, unpublished

[50] N. Kurahashi, G. Gratta Phys.Rev. D78 (2008) 092001

[51] N. Kurahashi et al., Pys. Rev. D82, (2010) 073006

[52] S. Danaher, J. Phys.Conf. ser. 81, (2007) 012011

[53] S. Bevan, NIM A 604 (2009) 143

[54] S. Danaher et al., NIM A (2010), doi:10.1016/j.nima.2010.11.075

[55] g. Riccobene et al., NIM A 604 (2009) 149

[56] V. Aynutdinov et al., Astropart. Phys. 7 (1997) 263

[57] R. Lahmann, NIM A, doi:10.1016/j.nima.2010.11.157

[58] R. Lahmann, private communication

[59] K. Laihem, contribution to this proceedings 